\title{
SATISFAÇÃO DOS USUÁRIOS COM OS CUIDADOS DE ENFERMAGEM - REVISÃO INTEGRATIVA DA LITERATURA
}

\author{
Deolinda Bernardo ${ }^{1,2}$, Pedro Bernardes Lucas² \\ ${ }^{1}$ Agrupamento de Centros de Saúde de Cascais, Portugal. dcbernardo@esel.pt; \\ ${ }^{2}$ Escola Superior de Enfermagem de Lisboa, Portugal. prlucas@esel.pt
}

\begin{abstract}
Resumo. Introdução: A satisfação do usuário (SU) é um indicador significativo e uma das dimensões fundamentais para melhorar a qualidade dos cuidados de enfermagem (CE). Os enfermeiros detêm um papel extremamente importante na SU com os cuidados de saúde, uma vez que estes constituem a maioria dos profissionais de saúde nas diferentes equipas e contactam diária e frequentemente com os usuários. Objetivo: Analisar o nível de satisfação dos usuários face aos cuidados de enfermagem em CSP. Métodos: Revisão integrativa da literatura com 3 etapas. 1) Uma pesquisa inicial na CINHAL e MEDLINE. 2) Uma pesquisa mais alargada, utilizando as mesmas palavras-chave e termos de pesquisa, nas restantes bases de dados da plataforma EBSCOHost. 3) Pesquisa nas referências bibliográficas dos artigos selecionados. Os estudos selecionados foram do período entre 2009 e 2019. Resultados: Selecionaram-se vinte estudos que relatam achados sobre as dimensões que constituem o conceito de SU: arte no atendimento; qualidade técnica ou científica; custos; organização física e ambiental; disponibilidade dos profissionais; continuidade de cuidados; e resultados dos CE. Conclusão: A SU é descrita como o valor e reação aos CE que receberam. A SU com os CE é considerada como indicador fundamental da qualidade dos serviços de saúde.
\end{abstract}

Palavras-chave: Satisfação do Paciente; Cuidados de Enfermagem; Qualidade da Assistência à Saúde; Gestão em Enfermagem.

\section{PATIENT SATISFACTION WITH NURSING CARE - INTEGRATED REVIEW}

Abstract. Introduction: User satisfaction (US) is a significant indicator and one of the fundamental dimensions to improve the quality of nursing care (NC). Nurses play an extremely important role in PS with health care, since they make up the majority of health professionals in the different teams and contact daily and frequently with patients. Goals: To analyze about US with NC in Primary Health Care. Methods: Integrative literature review with 3 steps. 1) An initial search at CINHAL and MEDLINE. 2) A broader search, using the same keywords and search terms, in the remaining databases of the EBSCOHost platform. 3) Search the bibliographic references of the selected articles. The selected studies were from the period between 2009 and 2019. Results: Twenty studies were selected that report findings on the dimensions that make up the US concept: art in care; technical or scientific quality; costs; physical and environmental organization; availability of professionals; continuity of care; and CE results. Conclusions: US is described as the value and reaction to the NC they received. US with NC is considered a fundamental indicator of the quality of health services.

Keywords: User Satisfaction; Nursing Care; Quality of Health Care; Nursing Management.

\section{INTRODUÇÃO}

A satisfação do usuário é um indicador significativo da qualidade dos cuidados prestados. Os enfermeiros procuram um exercício profissional de excelência conforme os padrões de qualidade dos cuidados de enfermagem, para tal acontecer é imperativo pesquisar a qualidade dos cuidados na perspetiva do usuário. 
Os enfermeiros detêm um papel extremamente importante na satisfação do usuário com os cuidados de saúde, uma vez que estes constituem a maioria dos profissionais de saúde nas diferentes equipas e contactam diária e frequentemente com os usuários.

$\mathrm{Na}$ área da enfermagem, a satisfação dos usuários resulta da relação entre as expectativas dos usuários relativamente aos cuidados de enfermagem e a perceção dos cuidados efetivamente recebidos. Por isso, Ribeiro (2003) refere que "os consumidores de cuidados de saúde requerem cada vez mais cuidados de qualidade, sendo que um dos indicadores da qualidade desses cuidados é a satisfação dos usuários" e “... é de extrema importância ser capaz de definir, medir e avaliar a qualidade dos cuidados de saúde prestados, a fim de manter e aumentar a satisfação do usuário" (Johansson, Oléni \& Fridlund, 2002, p.337).

Conhecer a satisfação dos usuários acerca dos cuidados de enfermagem, pontos fortes e fracos, potencialidades e deficiências permite aos gestores alguns caminhos decisórios para a reorganização das atividades assistenciais, de gestão e de ensino, conduzindo a uma melhoria contínua da qualidade dos cuidados.

No contexto da saúde, a satisfação dos usuários é uma das variáveis essenciais a incluir nos modelos de gestão e governo da qualidade dos serviços. (Vilela 2018).

Deste modo o objetivo desta revisão é analisar o nível de satisfação dos usuários face aos cuidados de enfermagem em cuidados de saúde primários (CSP), tendo por base a seguinte questão de revisão: "Qual o nível de satisfação dos usuários face aos cuidados de enfermagem prestados pelas equipas dos CSP?"

\section{MÉTODOS}

Esta revisão teve como referência a metodologia proposta por Whittemore \& Knafl (2005) para revisões integrativas: tendo como objetivo "analisar a evidência científica acerca da satisfação dos usuários face aos cuidados de enfermagem". A questão de revisão é: Qual o nível de satisfação dos usuários face aos cuidados de enfermagem prestados pelas equipas dos CSP?

A pesquisa considerou os idiomas português, inglês e espanhol e delimitou-se a artigos publicados entre 2009 e 2019, visto compreender um recorte de tempo mais próximo de toda a realidade. No entanto pela dificuldade em encontrar artigos de estudos realizados em cuidados de saúde primários foram elegíveis cinco artigos de 2001 a 2019. 
Elegeu-se a plataforma EBSCOhost e selecionaram-se as bases bibliográficas eletrónicas: CINAHL, MEDLINE, Cochrane.

Identificámos quatro descritores: Satisfação dos usuários (User satisfaction); Cuidados de Enfermagem (Nursing Care); Qualidade dos cuidados de enfermagem (Quality of health care); Gestão dos cuidados de enfermagem (Nursing care management), validados através dos Descritores em Ciências da Saúde - DeCS (compatível com Medical Subject Headings $\mathrm{MeSH}$ ). Estes foram combinados através das expressões booleanas OR e AND da seguinte forma: User Satisfaction and (Nurse management OR Nurse Leaders OR Nurse managers) and nurs* and Quality of Health Care.

Como critérios de inclusão considerou-se: artigos apresentados com texto integral; referentes a cuidados de enfermagem; apresentarem informação ao nível dos parâmetros considerados imprescindíveis de analisar: Participantes (usuários ou pacientes, enfermeiros (que exercem funções em cuidados de saúde primários), Intervenções (estudos que se reportam a cuidados de enfermagem), Comparações (quando existentes), Resultados (perceções sobre a satisfação dos usuários face aos cuidados de enfermagem) e desenho do estudo e ainda, ano e autor(es). Como critérios de exclusão considerou-se artigos não primários, como os de opinião e as revisões, e aqueles que, após a leitura na íntegra, não respondessem ao objetivo da revisão.

A Figura 1 especifica os resultados das etapas da análise.

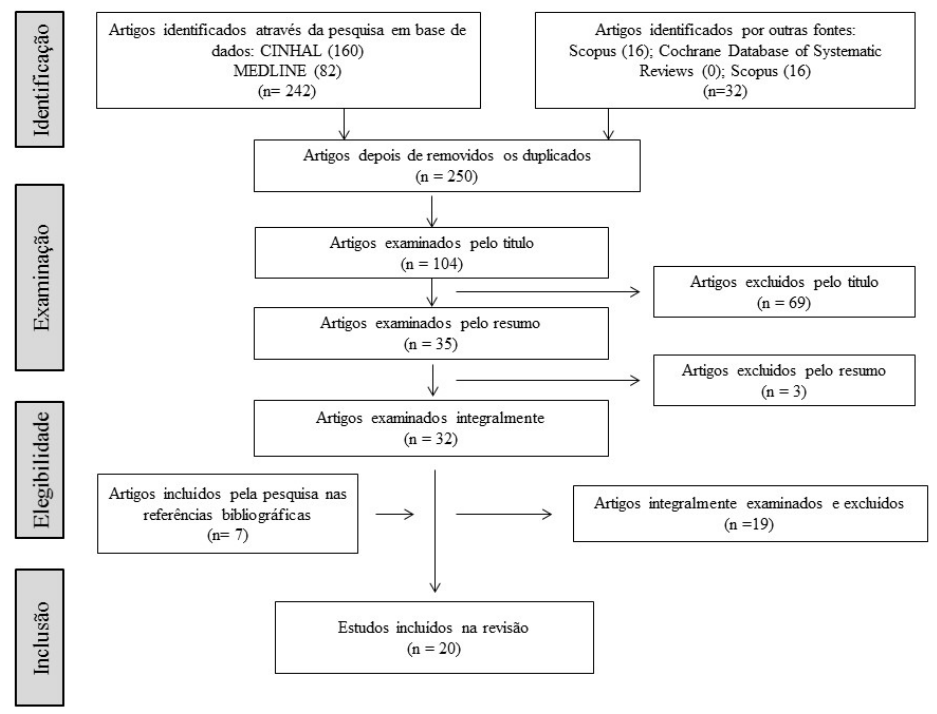

Figura 1. Fluxograma do processo de pesquisa e seleção dos estudos 
A seleção dos estudos foi realizada por meio da leitura minuciosa dos títulos e resumos, de modo que foram para a seleção final os estudos que atendiam aos critérios de inclusão supracitados. Para a seleção final dos artigos foi realizada a leitura do trabalho na íntegra, sendo selecionados aqueles cujos tópicos de interesse abordados são: autor, ano de publicação, idioma ou país de origem da publicação, título do artigo, tipo de estudo e população abrangida. Objetivo da investigação, resultados encontrados e implicação para a gestão em enfermagem.

\section{DISCUSSÃO DOS RESULTADOS}

Estes estudos demostram que a satisfação do usuário é o valor e reação aos cuidados de enfermagem que receberam. A satisfação do usuário com os cuidados de enfermagem é considerada como indicador fundamental num serviço de saúde de qualidade.

É multidimensional o conceito de satisfação por parte dos usuários e aborda os seguintes aspetos: a arte no atendimento, a qualidade técnica ou científica, o custo, a organização física e ambiental, a disponibilidade dos profissionais, a continuidade de cuidados e resultados. Medir o nível de satisfação do usuário é um desafio e deve ser feito com precisão a reação do usuário aos cuidados que recebeu usando um instrumento válido e confiável. Medir a satisfação do usuário com diferentes instrumentos pode dar resultados diferentes do resultado (nível de satisfação) (Margolis et al, 2003; Vilela, 2018).

\subsection{Qualidade técnica ou científica}

Assim, medir o nível de satisfação do usuário com os cuidados de enfermagem é importante para determinar a satisfação geral do serviço de saúde prestado e avaliar as necessidades e expectativas dos usuários pode ajudar os enfermeiros a planear intervenções para com estes (Atallah, Hamdan-Mansour, Al-Sayed \& Aboshaiqah, 2013; Karaca \& Durna, 2019; Wai, Chi-Yang, Wen, 2013).

A satisfação do usuário é uma das principais preocupações do sistema de saúde, particularmente nos países em desenvolvimento. Usuários satisfeitos são mais propensos a ter um bom relacionamento com enfermeiros, o que sugere melhora da qualidade de cuidado. A literatura também sugeriu que o usuário estando satisfeito está diretamente ligada a melhores resultados para os próprios (Alhusban \& Abualrub, 2009; Atallah et al, 
2013; Fan, Burman, McDonell \& Finn, 2005; Freitas, Silva, Minamisava, Bezerra \& Sousa, 2014).

Além disso, alcançar o nível ideal de satisfação do usuário com os cuidados de enfermagem resulta em melhor adesão do usuário ao regime de cuidados de saúde (Johansson, Oléni \& Fridlund, 2002).

A satisfação do usuário com os cuidados de enfermagem, podem ser afetados por inúmeros fatores, incluindo fatores relacionados com o usuário (por exemplo, residência, história de hospitalização prévia) e fatores relacionados com o contexto (por ex. disponibilidade do enfermeiro/s designados, comportamentos dos enfermeiros e ambiente físico circundante) (Ahmed, Shehadeh \& Collins, 2013; Batbaatar, Dorjdagva, Luvsannyam, Savino \& Amenta, 2016; Gill \& White, 2009; Santos, Sardinha \& Santos, 2017).

A avaliação da satisfação do usuário tem sido para as instituições de saúde como uma estratégia para compreender os fatores que influenciam a perceção da qualidade do cuidado, na perspetiva do usuário (Alhusban \& Abualrub, 2009; Crow et al, 2003; Karaca \& Durna, 2019).

A satisfação do usuário é avaliada como o grau de coerência entre as expectativas e a perceção do indivíduo sobre o cuidado recebido (Crow et al, 2003; Johansson, Oléni \& Fridlund, 2002) que reflete a avaliação cognitiva e emocional do usuário com base em experiências anteriores (Mulugeta, Wagnew, Dessie, Biresaw \& Habtewold, 2019).

Também pode ser compreendida como o grau em que os cuidados de enfermagem atendem às expectativas do usuário em termos da arte do cuidado, da qualidade técnica, do ambiente físico, da continuidade de cuidados prestados e eficácia dos resultados. Entre os fatores que influenciam a satisfação do usuário com os cuidados de enfermagem destacamse aqueles que envolvem o relacionamento entre enfermeiro e usuário (Atallah, HamdanMansour, Al-Sayed \& Aboshaiqah, 2013; Karaca \& Durna, 2019; Oliveira, 2012) o apoio afetivo, as informações sobre a saúde, o controle da decisão pelo usuário e a competência técnica do profissional que o assiste (Ahmed, Shehadeh \& Collins, 2013; Johansson, Oléni \& Fridlund, 2002; Wai, Chi-Yang, Wen, 2013). 
Os estudos da satisfação do usuário passam por ser sobre a avaliação da perceção do usuário sobre a qualidade dos cuidados prestados e tem de ser as organizações a concentrar-se na qualidade percebida pelos usuários (Johansson et al, 2002).

Para realizar as avaliações da satisfação dos usuários face aos cuidados de enfermagem, recomenda-se a utilização de instrumentos com confiabilidade e validade reconhecidos (Johansson et al, 2002).

A avaliação da satisfação dos usuários deve ser pelo uso de ferramentas que garantam uma qualidade útil para o desenvolvimento de cuidados de enfermagem de qualidade (Margolis et al, 2003).

Nos últimos anos, muitos estudos foram realizados para determinar o nível de satisfação do usuário com os cuidados de enfermagem. Por exemplo, estudos feitos na Malásia (Wai et al, 2013), Brasil (Freitas et al, 2014; Santos et al, 2017) e Arábia (Atallah et al, 2013) mostraram que, a satisfação do usuário com os cuidados de enfermagem é alta.

A avaliação da satisfação do usuário possibilita ao enfermeiro gestor implementar mudanças e propor ações para melhoria da qualidade dos cuidados prestados (Freitas, Parreira \& Domingues, 2016; Johansson et al, 2002; Wai et al, 2013) contribuindo para a visibilidade do trabalho da equipa de enfermagem nas instituições de saúde (Wai et al, 2013).

Quanto menor o grau de escolaridade, maior o nível de satisfação do usuário (Ahmed et al, 2013). Este resultado é coerente com os descritos em outras pesquisas, que indicam a escolaridade como um fator que influencia na utilização de serviços de saúde.

Reformular as políticas de cuidados de enfermagem de saúde comunitária para dar mais atenção ao aumento do nível de coordenação e aos aspetos interpessoais do cuidado prestado provavelmente aumentaria sua satisfação (Ahmed et al, 2013).

Os gestores em enfermagem precisam manter os cuidados de enfermagem de qualidade e desenvolver estratégias para melhorar esses mesmos cuidados, não tornando a linguagem como barreira, utilizando habilidades para a disseminação de informação e assim melhorar a satisfação dos usuários (Atallah et al, 2013). 
A satisfação do usuário passa por ser sobre a avaliação da perceção do usuário sobre a qualidade dos cuidados prestados e tem de ser as organizações a concentrar-se na qualidade percebida pelos usuários (Gill \& White, 2009).

Uma implicação importante para futuras pesquisas é continuar a elucidar os fatores que influenciam a satisfação do usuário para com os cuidados de enfermagem, na perspetiva do usuário (Johansson et al, 2002).

No estudo de Karaca \& Durna (2019), os enfermeiros precisavam de mostrar maior interesse para com os usuários. A avaliação da satisfação dos usuários deve ser pelo uso de ferramentas que garantam uma qualidade útil para o desenvolvimento de cuidados de enfermagem de qualidade (Margolis et al, 2003).

\subsection{Arte no atendimento/Disponibilidade dos profissionais}

As características sociodemográficas dos usuários como idade, sexo, nível de escolaridade (Santos, Sardinha \& Santos, 2017) e experiência anterior de internamento anteriores (Mulugeta et al, 2019), também têm sido apontadas como variáveis que exercem influência na satisfação do usuário.

A equipa de enfermagem ocupa uma posição de destaque em influenciar a satisfação do usuário com o cuidado recebido, pois é a responsável pelo cuidado direto ao usuário, pela organização do cuidado e pela coordenação do trabalho de enfermagem. Os Enfermeiros são um grupo profissional fundamental do sistema de saúde, que passa mais tempo com os usuários e fornecem cerca de $80 \%$ do serviço de cuidados de saúde.

É importante destacar que a informação dada pelos enfermeiros constitui um dos fatoreschave para a satisfação quanto ao cuidado de enfermagem recebido (Johansson et al, 2002) e também para a atuação do enfermeiro no processo de alta hospitalar, pois o aspeto educacional é imprescindível para garantir o autocuidado do usuário e até evitar possíveis reinternamentos decorrentes da falta de orientação (Atallah et al, 2013).

Os usuários ao serem considerados em relação ás suas expectativas, apresentam melhores condições de responder positivamente às intervenções terapêuticas (Atallah et al, 2013; Johansson et al, 2002; Santos et al, 2017), pois envolvem- se com o cuidado prestado e aderem melhor às orientações propostas. 


\subsection{Continuidade de cuidados}

Com o foco nos cuidados de enfermagem, o estudo de Chaves et al, (2016) mensurou o grau de satisfação dos pacientes das variadas unidades de CSP como "muito bom" (97,3\%). Em relação às consultas realizadas pelos enfermeiros, a maioria $(51,9 \%)$ respondeu ser pertinente a sua realização.

A satisfação e a qualidade, estão presentes em vários estudos, podendo ser entendidas por visões diferentes, como sejam a do enfermeiro que presta cuidados, a do enfermeiro gestor, a do usuário e ainda a da direção da organização.

Conhecer a satisfação dos usuários acerca dos cuidados de enfermagem, pontos fortes e fracos, potencialidades e deficiências permite aos gestores alguns caminhos decisórios para a reorganização das atividades assistenciais, de gestão e de ensino, conduzindo a uma melhoria contínua da qualidade dos cuidados.

A opinião dos usuários sobre suas expectativas e satisfação com os cuidados de enfermagem pode ser considerada uma importante oportunidade para o enfermeiro planear e implementar estratégias adequadas que melhorem a qualidade dos cuidados de enfermagem (Abdel Maqsood, Oweis \& Hasna, 2012).

A satisfação do utente é decisiva para a qualidade e eficiência dos cuidados prestados, sendo necessário o compromisso de todos os prestadores na implementação de práticas sistemáticas de gestão que conduzam à satisfação, dando particular atenção à melhoria contínua dos processos organizacionais (Chaves et al, 2016).

Melhores informações sobre os fatores que afetam a satisfação ajudarão os prestadores de serviços de saúde e gestores a melhorar a qualidade do serviço prestado. Orientação sobre métodos de colheita de resultados dos usuários irá garantir informações confiáveis para o processo de tomada de decisão (Crow et al, 2003).

A melhoria continua da qualidade dos cuidados de enfermagem pode melhorar a satisfação do usuário bem como traz benefícios à organização (Fan, Burman, McDonell, Fihn, 2005).

Há necessidade de a instituição centrar os seus objetivos num sistema de avaliação permanente da qualidade do cuidado, visando o atendimento das expectativas dos usuários (Freitas, Parreira \& Domingues, 2016). 
Conhecer o índice de satisfação dos utentes que recorrem aos serviços de saúde é um elemento estruturante e fundamental para a eficiência e qualidade dos cuidados prestados (Oliveira, 2012).

\section{CONCLUSÕES}

A satisfação é influenciada diretamente pela qualidade dos cuidados prestados, pelo profissionalismo, pela acessibilidade e pela qualidade técnica e eficiência, pelo valor percebido, pelo envolvimento, pela imagem e pela equidade.

A satisfação do usuário é decisiva para a qualidade e eficiência dos cuidados de enfermagem prestados, sendo necessário o compromisso de todos na implementação de práticas organizadas de gestão que conduzam à satisfação, dando particular atenção à melhoria contínua dos processos organizacionais. A avaliação da satisfação do usuário temse revelado uma ferramenta indispensável para a melhoria dos cuidados de saúde.

Existem implicações para a gestão em enfermagem, devendo estes conhecer os aspetos positivos e negativos da qualidade dos seus serviços, procurar definir aspetos negativos observados pelos usuários e deste modo procurar minimizar as falhas no sistema de prestação de cuidados através da análise das razões subjacentes ás mesmas. Apesar dos esforços em minimizar estas situações, as falhas nos cuidados não podem ser totalmente evitadas, pelo que os gestores devem estar preparados para a ocorrência e resolução destes problemas.

Os gestores em enfermagem devem adotar medidas que despertem a cooperação, a satisfação e a partilha de informação, podendo lançar medidas coletivas catalisadoras destes fatores.

\section{REFERÊNCIAS}

Abdel Maqsood, A. S., Oweis, A. I., \& Hasna, F. S. (2012). Differences between patients' expectations and satisfaction with nursing care in a private hospital in Jordan. International Journal of Nursing Practice, 18(2), pp 140-146. doi:10.1111/j.1440-172X.2012.02008.x

Ahmed, M., Shehadeh, A. \& Collins, M. (2013). Quality of nursing care in community health centers: clients' satisfaction. Health Science Journal, 7(2): pp 229-36. http://hdl.handle.net/11400/1412

Alhusban, M. A. \& Abualrub, R. F. (2009). Patient satisfaction with nursing care in Jordan. Journal of Nursing Management. Vol. 17. Pp 749-75817(6):749-58. doi:10.1111/j.1365-2834.2008.00927.x 
Atallah MA, Hamdan-Mansour AM, Al-Sayed MM, Aboshaiqah AE. (2013). Patients' satisfaction with the quality of nursing care provided: the Saudi experience. International Journal of Nursing Practice, 19(6). 584-90. https://doi.org/10.1111/ijn.12102

Batbaatar, E., Dorjdagva, J., Luvsannyam, A., Savino, M. M., \& Amenta, P. (2016). Determinants of patient satisfaction: a systematic review. Perspectives in Public Health, 137(2). 89-101. https://doi.org/10.1177/1757913916634136

Chaves, C.; Duarte, J.; Amaral, O. et al. (2016). Satisfação dos utentes dos cuidados de saúde primários com os cuidados de enfermagem - amostra da Região Centro de Portugal. International Journal of Developmental and Educational Psychology, 1 (2). 339-346. https://doi.org/10.17060/ijodaep.2016.n2.v1.582

Crow, R., Gage, H., Hampson, S., Hart, J., Kimber, A. \& Storey, L. (2003). The measurement of satisfaction with health care: implications for practice from a systematic review of the literature. Health Technol Assessment. $6(32)$.

Fan, V.S., Burman, M., McDonell, M. B. \& Fihn, S. D. (2005). Continuity of care and other determinants of patient satisfaction with primary care. Journal of General Internal Medicine. DOI: 10.1111/j.15251497.2005.40135.x

Freitas, J. S., Silva, A. E., Minamisava, R., Bezerra, A. L., Sousa, M. R. (2014). Qualidade dos cuidados de enfermagem e satisfação do paciente atendido em um hospital de ensino. Revista Latino-Americana de Enfermagem. 22(3): 454-60. DOI: 10.1590/0104-1169.3241.2437

Freitas, M. J., Parreira, P. M. \& Domingues, J. P. (2016). Avaliação das propriedades psicométricas da Escala Satisfação dos Usuários com os Cuidados de Enfermagem no Hospital. Revista Enfermagem Referência. 4(10), 9-17. http://dx.doi.org/10.12707/RIV16031

Gill, L. \& White, L. (2009). A critical review of patient satisfaction. Leadership in Health Services; 22: pp 8-19. DOI $10.1108 / 17511870910927994$

Johansson, P., Oléni, M., \& Fridlund, B. (2002). Patient satisfaction with nursing care in the context of health care: a literature study. Scandinavian Journal of Caring Sciences, 16(4), pp 337-344.

Karaca, A., \& Durna, Z. (2019). Patient satisfaction with the quality of nursing care. Nursing Open; 6:535-545. https://doi.org/10.1002/nop2.237

Margolis, S. A., Al-Marzouq, S., Revel, T. \& Reed, R. L. (2003). Patient satisfaction with primary health care services in the United Arab Emirates. International Journal for Quality in Health Care. 15(3). 241-9. DOI: 10.1093/intqhc/mzg036

Mulugeta, H., Wagnew, F., Dessie, G., Biresaw, H. \& Habtewold, T. (2019). Patient satisfaction with nursing care in Ethiopia: A systematic review and meta-analysis. BMC Nursing. Vol 18. pp 18-27. https://doi.org/10.1186/s12912-019-0348-9

Oliveira, A. (2012). Satisfação dos utentes numa unidade de saúde do interior. Dissertação de mestrado, Instituto Universitário de Lisboa: ISCTE, Lisboa. Escola Superior de Enfermagem do Porto, Portugal.

Rafill, F., Hajiezhad, M. \& Haghani, H. (2009). Nurse caring in Iran and its relationship with patient satisfaction. Australian Journal of Advanced Nursing. 26(2). 75-84.

Ribeiro, A. L. A. (2003). Satisfação dos Utentes com os Cuidados de Enfermagem: Construção e validação de um instrumento de medida. Porto: Escola Superior de Enfermagem São João. Dissertação para concurso de Provas Públicas para Professor Coordenador na área Científica de Ciências de Enfermagem.

Ribeiro, R. M. (2014). Cuidados de Enfermagem prestados pelas Equipas de Cuidados Continuados Integrados Satisfação dos Utentes e Cuidadores. Dissertação de mestrado. Escola Superior de Enfermagem Porto. 
Santos, M. A., Sardinha, A. H. L. \& Santos, L. N. (2017). Satisfação dos usuários com os cuidados dos enfermeiros. Revista Gaúcha de Enfermagem. 38(1).

Silva, E. (2013) Ganhos em satisfação face aos cuidados de enfermagem de reabilitação: Doentes dependentes. Dissertação de mestrado. Escola Superior de Enfermagem do Porto, Portugal.

Vilela, C. (2018). Impacte do modelo de implementação das Equipas de Cuidados Continuados Integrados: Satisfação dos usuários com os cuidados de enfermagem. Tese para obtenção de Doutoramento. Porto.

Wai, M. T., Chi-Yang, S. \& Wen, C. L. (2013). Patient Satisfaction with Nursing Care: A Descriptive Study Using Interaction Model of Client Health Behavior. International Journal of Nursing Science. 3(2): pp 51-56. DOI: 10.5923/j.nursing.20130302.04

Walsh, M. \& Walsh, A. (2001). Measuring patient satisfaction with nursing care: experience of using the Newcastle Satisfaction with Nursing Scale. Journal of Advanced Nursing, 29(2), 307-315.

Whittemore, R. \& Knafl, K. (2005). The integrative review: updated methodology. Journal of Advanced Nursing, 52(5), 546-553. https://doi.org/10.1111/j.1365-2648.2005.03621.x 